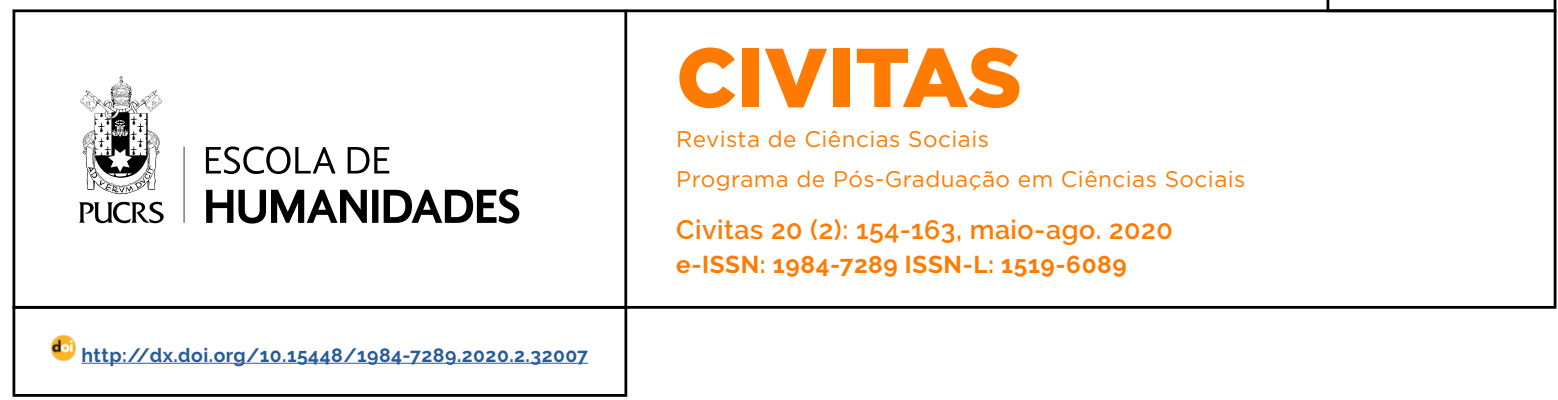

DOSSIÊ: MEDIO AMBIENTE EN DISPUTA

\title{
La efectuación de los cuerpos en dos conflictos ambientales en Argentina: afectados ambientales y discursos de verdad
}

\author{
A produção dos corpos em dois conflitos ambientais na Argentina: afetados \\ ambientais e discursos de verdade \\ The production of bodies in two environmental conflicts in Argentina: environmental \\ affects and truth speeches
}

Erika Saccucci $^{1}$
orcid.org/0000-0002-2469-0988
erika_sac34@hotmail.com

Recebido em: 20 set. 2018. Aprovado em: 09 ago. 2019. Publicado em: 4 ago. 2020.
Resumen: En este artículo hemos estudiado el conflicto que se produce en torno a los discursos científicos como discursos de poder en dos conflictos ambientales en la ciudad de Córdoba, Argentina- Pueblos Unidos y Vudas contra la empresa Porta. Se ha trabajado con 20 entrevistas en profundidad combinando técnicas del análisis de contenido y análisis de discurso. En el análisis emergen dos efectuaciones corporales: por una parte, la de afectados ambientales que refiere a los cuerpos contaminados y a la conflictiva relación con el discurso científico que, articulado con los intereses del capital no da cuenta de la afección que presentan los cuerpos. Por otra parte, se reivindica la existencia de cuerpos sanos, libres de contaminación y se denuncia al discurso científico por manipular los estudios, a los fines de sostener la idea de contaminación. En conclusión, el discurso científico funciona como discurso de poder que atraviesa las luchas. La diversa configuración de cuerpos en cada caso se explica como efectuación de la singular articulación que se ha producido entre las diversas fracciones del capital y el discurso científico. Palabras clave: Córdoba. Cuerpos. Contaminación. Dispositivos.

Abstract: In this article we have studied the conflict around scientific discourses as power speeches in two environmental conflicts in the city of Cordoba, Argentina Pueblos Unidos and Vudas against Porta company. We have worked with 20 in depth interviews combining content analysis and discourse analysis techniques. From the analysis emerge two body configurations: First, environmental affected that refers to the contaminated bodies and to the conflictive relation with the scientific discourse that, articulated with the interests of capital does not account for the affliction presented by the bodies. Second, the existence of healthy bodies, free of pollution and the denunciation of scientific discoursed for manipulating the studies in order to sustain the idea of pollution. In conclusion, the scientific discourse functions as a discourse of power that goes through the struggles. The different configuration of bodies in each case is explained as an effectuation of the singular articulation that has occurred between the different fractions of capital and the scientific discourse. Keywords: Córdoba. Bodies. Contamination. Devices of power.

Resumo: Neste artigo temos estudado o conflito que se produz em torno dos discursos científicos como discursos de poder em dois conflitos ambientais na cidade de Córdoba, Argentina - Pueblos Unidos e Vudas contra a empresa Porta. Trabalhou-se com 20 entrevistas em profundidade combinando técnicas da análise de conteúdo e análise de discurso. Na análise emergem duas configurações corporais: por um lado, a de afetados ambientais que refere aos corpos contaminados e à conflituosa relação com o discurso científico que, articulado com os interesses do capital, não dá conta da condição que apresentam os corpos. Por outro lado, reivindica-se a existência de corpos sãos, livres de contaminação e denuncia-se ao discurso científico por manipular os estudos, a fim de sustentar a ideia de contaminação. Em conclusão, o discurso científico funciona como
Artigo está licenciado sob forma de uma licença Creative Commons Atribuição 4.0 Internacional. 
discurso de poder que atravessa as lutas. A diferente configuração de corpos em cada caso explica-se como efetuação da singular articulação que se produziu entre as diversas frações do capital e o discurso científico.

Palavras-chave: Córdoba. Corpos. Contaminação. Dispositivo.

\section{Introducción}

Existe una dimensión social y cultural de los problemas ambientales (Douglas y Wildavsky 1982), que posee el mismo estatuto de realidad que la derivada de su dimensión física. Esta forma de existencia de los problemas depende de un proceso social de construcción y tiene que ver con su percepción, significación y reconocimiento público. Los sujetos desarrollan mecanismos por medio de los cuales determinados aspectos de la realidad adquieren significado colectivo especial, que los hace surgir en el escenario público como conflictos (Lezama 2001).

De acuerdo a Auyero y Swistun (2008) el sufrimiento ambiental hace sentido en tanto experiencia individual, ya que el padecimiento es parte de lo vivenciado por el cuerpo, pero a la vez es social ya que las situaciones de sufrimiento son construcciones colectivas ancladas en contextos relacionales y discursivos que moldean culturalmente las formas en las que los actores viven y entienden su dolor y las causas que lo producen. El sufrimiento ambiental debe ser entendido como una forma particular de sufrimiento social causado por las acciones contaminantes concretas de actores especificos. En otras palabras, el sufrimiento ambiental no es experimentado en aislamiento, sino dentro de contextos discursivos y en relaciones de poder. Estos contextos dan forma a los caminos en los cuales la aflicción es vivida y comprendida.

Entonces, el cuerpo en los conflictos por el ambiente, asume una importancia central no solo como "superficie" expuesta a la posible contaminación sino que a su vez encarna los procesos sociales de significación sobre la afección (Orellana 1999; Sabatini 1997). En este trabajo no proponemos una perspectiva objetivista del cuerpo sino una que centre su mirada en la intersubjetividad que lo significa. Aquello que interesa conocer en los dos casos estudiados es cómo han significado los discursos cientificos sobre contaminación. En esa significación, el cuerpo es fundamental, en tanto destinatario de los discursos científicos pero al mismo tiempo como cuerpo vivido, como experiencia cotidiana que le brinda a los sujetos indicios propios sobre lo que los discursos médicos sostienen.

En este artículo se han estudiado dos conflictos por el ambiente: Pueblos Unidos y Vudas. En ellos se construyen dos sentidos opuestos sobre el discurso científico: en Pueblos Unidos aparece como un dispositivo de poder cuya efectuación en la ficción de cuerpos enfermos mientras que en el discurso de Vudas contra la fábrica Porta Hnos. el discurso científico es aquel que debe demostrar las enfermedades presentes en la comunidad barrial y su vínculo con la contaminación.

Pueblos Unidos es una toma de tierra que se encuentra emplazada sobre un ex basural a cielo abierto. Este caso presenta una interesante discusión en torno a la (in)existencia de contaminación y respecto de lo que se considera vivible/saludable. En línea opuesta, el caso Vudas contra la empresa Porta Hermanos pone en cuestión la calidad ambiental a partir de afirmar la presencia de contaminación y la creciente presencia de enfermedades. Contraponer estos dos casos resulta interesante ya que en el primero los vecinos centran su lucha en la conservación de la tierra, desestimando la presencia de contaminación; mientras que, en el segundo, las vecinas de Vudas insisten, desde 2012, en la insalubridad que supone la permanencia de la planta productora de alcohol y bioetanol. En definitiva, el criterio de selección de los casos ha sido la consideración del rol fundamental que ha desempeñado el discurso científico en estos conflictos asumiendo sentidos opuestos: en uno afirma la contaminación y en otro la niega.

Hemos propuesto que los conflictos son instancias de visibilización de las luchas sociales y que en el estudio de la conflictividad es donde se hace posible conocer con mayor detalle la operatoria del poder y la agencia de los sujetos. Para conocer la operatoria del poder hemos propuesto el concepto de dispositivos de poder. Particularmente, nos hemos centrado en la lucha 
activa que se produce en torno a los discursos científicos en tanto discursos de poder.

A continuación, se exponen las herramientas conceptuales desde las cuales se ha trabajado y las principales decisiones metodológicas. Luego, se presentan dos efectuacioness de cuerpos que se desprenden del análisis de las entrevistas: afectados ambientales y cuerpos libres de contaminación. Finalmente, se han presentado las conclusiones, donde señalamos que esta diversa configuración de los cuerpos en cada caso, refiere al modo en el cual el discurso cientíico se ha relacionado con las diversas fracciones del capital, favoreciendo sus intereses. El discurso científico, en tanto dispositivo de poder y discurso de verdad configura diversas realidades y con ellas cuerpos.

\section{Los discursos científicos como dispositivos de poder}

En los conflictos por el ambiente los discursos científicos emergen como dispositivos de poder fundamentales. Los dispositivos de poder organizan la experiencia del presente, pues regulan las posibilidades de agencia y los límites para la acción ya que fijan fronteras a los comportamientos posibles. Su operatoria se despliega como un campo de posibilidades que incitan y/o propician determinadas decisiones y acciones, al tiempo que inhiben y/o prohiben otras. Así, los dispositivos se configuran en la unión de elementos heterogéneos: discursos, instituciones, diseños arquitectónicos, decisiones reglamentarias, leyes, medidas administrativas, enunciados científicos, proposiciones filosóficas, morales, entre otras que puedan configurarse y articularse (Foucault 1994) destinadas a la regulación y control de las acciones de los sujetos.

Un dispositivo se caracteriza por (a) definir una serie de conexiones intimas entre saber y poder; (b) establecer la dispersión del poder a través de múltiples técnicas (la vigilancia, el castigo, el examen); (c) se trata de un conjunto heterogéneo que incluye diversos elementos: discursos, instituciones, edificios, leyes, medidas policiacas, proposiciones filosóficas; (d) siempre tiene una función concreta inscrita en una relación de poder.
En definitiva, la doble dinámica de hacer posible y de control con que operan los dispositivos, habilita un juego de acciones y decisiones discrecionales. Así pues, los dispositivos son, por un lado, marcos que permiten la creatividad e innovación que requiere la sociedad presente (Guattari y Rolnik 2005) y, por el otro, establecen los márgenes de las acciones y decisiones posibles de modo tal que las mismas sean manejables y gobernables (Ciuffolini 2010). Pero, al mismo tiempo, frente al despliegue de los dispositivos de poder se configuran resistencias (Murrillo 2004). El concepto de efectuación (Deleuze 1994) supone entender las configuraciones que se producen por las relaciones de poder como efectos siempre inacabados: es en esa tensión entre la fuerza configuradora del poder y las resistencias que se le oponen, donde reside como posibilidad, la agencia. Proponer desde esta perspectiva el abordaje implica reconocer su efectuación cotidiana recuperando la potencia creadora de los sujetos.

\section{Los casos estudiados y las decisiones metodológicas}

A los fines de esta investigación se han seleccionado dos conflictos en donde se presentan discusiones vinculadas a las condiciones medioambientales: Pueblos Unidos y Vecinos Unidos en Defensa de un Ambiente Seguro - Vudas - en contra de la permanencia de la empresa Porta Hnos.

\section{El caso de Pueblos Unidos}

Pueblos Unidos es una toma de tierra y es también conocida como Nuestro Hogar IV, data del año 2007 cuando diversas familias se organizaron para avanzar sobre tierras disponibles a los fines de resolver sus necesidades habitacionales. Estas tierras habían sido un basural a cielo abierto en los años 70 y aún presentaban grandes cantidades de residuos. La toma de tierra se desarrolló sin mayores dificultades y las y los vecinos avanzaron sobre la construcción en material de sus viviendas, en el trazado de calles de acuerdo a las disposiciones urbanas y en la lucha por los servicios. En 2009 se inicia un 
proceso conflictivo, a partir de la intervención de diversos niveles gubernamentales que sostenian que la tierra se encontraba contaminada como resultado de su función anterior como basural.

En diciembre de 2010 el Consejo Deliberante de la ciudad declaró a Pueblos Unidos como "inhabitable" a través de una ordenanza. Esta declaración resulta de fundamental importancia en tanto representa el primer caso en el país. Frente a esto, la comunidad barrial puede ser dividida entre quienes aceptan la idea de la presencia de contaminación pero carecen de otras opciones ${ }^{2}$ y quienes niegan la veracidad de estos estudios y sospechan que se busca "liberar" la tierra a los fines de construir un emprendimiento inmobiliario, o bien, destinarlo nuevamente para el enterramiento de basura y asi resolver de manera permanente la problemática estructural de los desechos de la basura de la ciudad. La resistencia de la mayoría de las y los vecinos a abandonar la tierra tomada dio inicio al conflicto por la tierra.

\section{El caso de Vecinos Unidos en Defensa de un Ambiente Seguro - Vudas- contra la fábrica Porta Hnos.}

Por su parte, la empresa Porta Hnos. es originariamente productora de alcohol, sin embargo, en el año 2012 incluyó dentro de sus actividades la producción de bioetanol, en un marco nacional de promoción y fomento de esta actividad. Así, el conflicto comenzó en 2012 cuando las y los vecinos escucharon sonidos de explosión provenientes de la fábrica. Alli se enteraron que la empresa Porta Hnos. había comenzado a producir bioetanol. Es importante señalar que esta empresa es la única planta productora de bioetanol localizada dentro del ejido urbano, en el corazón de un barrio residencial. ${ }^{3}$ Con el pasar del tiempo los olores nauseabundos y la aparición de múltiples afecciones como problemas respiratorios, alergias y problemas en la piel atrajeron la atención de las vecinas que comenzaron a informarse sobre las consecuencias de este tipo de producción.

La recurrencia de las enfermedades descriptas y la aparición de casos de cáncer, púrpura y abortos espontáneos las llevó a contactarse con Reduas (Red Universitaria de Ambiente y Salud) quienes realizaron un primer relevamiento de las afecciones de la zona. La encuesta detectó que 43,2 por ciento de los vecinos tiene cefaleas persistentes y 34,8 por ciento sufre de conjuntivitis y congestión ocular, por encima de la media parámetro. ${ }^{4}$ De alli que las vecinas organizadas en Vudas comenzaran a reclamar la relocalización de la planta hacia zonas no habitadas.

\section{Técnicas de recolección de datos y de análisis}

Para el estudio de estos conflictos se han realizado 20 entrevistas en profundidad a través de la implementación de la técnica de la bola de nieve. Para el análisis de los datos se han articulado herramientas del análisis de contenido y del análisis de discurso. Para ello, se diseñó una estrategia de aproximación consistente en varios momentos. En un primer momento se procedió al análisis de contenido de los datos generados durante el trabajo de campo para luego comenzar a indizar algunos elementos discursivos que pudiesen estar advirtiendo sobre posibles relaciones entre los conflictos analizados. En una segunda instancia se abordó cada caso específico a los fines de dar cuenta de sus particularidad y caracteristicas. Es decir, desarrollamos un trabajo en dos niveles o en dos momentos: el abordaje transversal de los casos a los fines de conocer las regularidades de la operatoria del poder y las formas de resistencia que producen los sujetos. A partir de ello construimos dimensiones analíticas que nos permitieron la

\footnotetext{
Es importante señalar que a los fines de esta investigación nos hemos centrado en aquellos discursos que niegan la existencia de contaminación en Pueblos Unidos. Sin embargo, también podemos encontrar discursos que asumen la presencia de contaminación pero relativizan sus efectos en un contexto de escasez de opciones para asegurar la vida. Estos últimos han sido abordados en "Estudio de la significación de la inseguridad ambiental en dos casos en Córdoba" (Saccucci 2019).

3 La empresa se encuentra radicada sobre avenida Valparaíso y la colectora de la Circunvalación, en Barrio San Antonio, el cual colinda con los barrios Inaudi, Posta de Vargas, Cañitas, 2 de Abril. Porta Hermanos instaló la planta en el año 1995. unas cuatro décadas después de la formación del barrio Parque San Antonio. Sin embargo, en ese momento la zona era categorizada como industrial. Posteriormente la categorización cambió y desde entonces la planta recibe extensiones gubernamentales en sus permisos de funcionamiento.

4 Colectivo de investigación el Llano en Llamas. 2013. Informe preliminar. Tomas de tierras en Córdoba. Disponible en: http://wwww.llanocordoba.com.ar/publicaciones/informes/345-toma-de-tierras-en-cordoba-informe-preliminar-mayo-2013-54949872. Recuperado: 15 mar. 2017.
} 
comparación de los casos. Un segundo momento donde cada caso fue abordado específicamente con el objetivo de identificar sus rasgos característicos que dan cuenta de la especificidad del despliegue del poder sobre cada conflicto. Este ejercicio posibilitó la sistematización de los datos y la comparación entre los casos.

\section{La efectuación de cuerpos: afectados ambientales y cuerpos libres de contaminación}

En los discursos de las/los entrevistados, el cuerpo asume una centralidad clave. Sobre él se asientan las reflexiones en torno a la existencia o no de contaminación en la tierra y el ambiente. Para el discurso científico, el cuerpo se convierte en objeto de estudio y observación a los fines de encontrar irregularidades y signos anómalos que indiquen la presencia de enfermedad y la consiguiente posibilidad de vincularlo a la existencia de contaminación. Así, mientras que en los discursos del caso Vudas el cuerpo aparece como aquello enfermo, en los del conflicto de Pueblos Unidos emerge un cuerpo sano y la enfermedad como ficción:

ER2: ¿Y por qué crees que les pueden haber mentido con los estudios? EO: A mi nunca me mostraron nada. Ni los plomos que tuvo la nena mia, que supuestamente tiene plomo, a mi no me mostraron nunca el papel. Y yo no me animé a preguntarle al médico si me iban a dar un papel o nada. Me dijeron nomás que tenía plomo, nada más. ER1: ¿Y sabés si a mucha gente le dijeron [...]? EO: A la mayoria le han dicho que tenian plomo. ER2: ¿Y la gente en general creyó lo que le estaban diciendo o se quedó con duda? EO: La mayoria de la gente se ha quedado con duda. Y a mi nunca me mostró nada. Y no sé como se enferman cuando tienen plomo. Nunca me explicaron cómo es el plomo. Pero a mi hija nunca se le cayó nada, ni un pelo se le ha caido. Ella anda bien, ella l...] ER2: claro, ino te dijeron cómo es! vos no sabés cómo darte cuenta si ella está bien, está mal... EO: No, ellos nunca me dijeron cómo se enferma [...]. Nunca me dijeron... lo único que me dijeron fue "Dale vitamina, dale esto, dale aquello". Tenia una caja de vitaminas, pero qué le voy a dar vitamina si no sé yo (Pueblos Unidos 20:27). 5
Esta cita expresa el conflicto en torno al discurso científico que sostiene que los cuerpos están contaminados. Este sostiene que muchos de los habitantes de Pueblos Unidos se encuentran enfermos al tener "plomo en sangre". Sin embargo, otros discursos lo ponen en duda ante la falta de pruebas que lo sustenten. Se construye, entonces, un hiato entre el saber científico, médico y la población al no poder transmitir de manera confiable los resultados de los estudios realizados. En la comunidad barrial esto se traduce en una desconfianza creciente sobre el discurso científico y sus usos y habilita otros relatos y saberes.

Así, el discurso científico es presentado como ajeno y se contrapone a lo que el propio cuerpo percibe/experimenta. Esta tensión se expresa en la polaridad "me dijeron" frente al "anda bien", es decir, que la autoconciencia del cuerpo tiene un peso superior a la palabra de un "otro" legitimado socialmente. La experiencia es lo que el cuerpo registra, aquello que se manifiesta y puede ser aprehendido por los propios sentidos. Esto se enuncia en el discurso de las y los entrevistados a través de los léxicos "nunca se le cayó nada", "ni un pelo se la ha caido". En este mismo sentido encontramos la siguiente cita:

EO: Bueno, antes decian que no construyamos, porque estas tierras son tierras contaminadas l... pero por lo menos, no pasa nada, está bien. ER2: Desde que se instaló tu hermano, nunca han tenido problemas. EO: Nunca hemos tenido problemas, encima desde que se organizó el barrio está mi hermano. Así, todo basural, limpiaron, aplanaron, todo. ER1: ¿Vos pensás que esta tierra está contaminada? EO: No creo, si en esta tierra corre cualquier [...] plantamos, da mejores frutos, da mejor, todo...como si esta tierra estuviera abonada, si hubiera tenido abono, algo, si las plantas están lindas...(Pueblos Unidos 25:15).

Aquí, el entrevistado pone en duda la presencia de contaminación al oponer la propia percepción. Este conocimiento "otro" ligado a lo popular se funda en la observación y valoración de los procesos naturales. Así, el crecimiento de las

\footnotetext{
5 Los códigos que se encuentran al final de los extractos de entrevista son identificatorios. El primer número refiere a la entrevista mientras que el segundo da cuentas del extracto. Ejemplo: (7:1) entrevista 7. cita 1
} 
plantas, su floración y sus frutos son presentadas como evidencias de un estado de la tierra que se aleja de lo peligroso. Por el contrario, sugiere una tierra en buenas condiciones para la vida natural y para el humano. El conocimiento popular se ancla en la percepción y experiencia. Experiencia en un sentido de lo vivencial, como algo vivido más que como una "concientización" a priori de la situación (Berger 2013).

Aquí, el cuerpo se coloca en un registro activo, el de quien observa, analiza y construye indicadores naturales que permiten concluir la inexistencia de contaminación y, por extensión, la ficción del cuerpo enfermo. Se denuncia, entonces, una construcción del cuerpo desde la enfermedad. Este relato del cuerpo enfermo habilita la producción de un consenso social que daría lugar a la desposesión de las tierras que estos colectivos han tomado y el avance del capital inmobiliario sobre las mismas.

Para el discurso científico, estas tierras se encuentran contaminadas por lo cual es preciso disminuir su contacto con los alimentos. Por el contrario, para la comunidad, el contacto con la tierra no sólo tiene una gran importancia en relación a sus tradiciones sino, además, implica probar las condiciones de la misma. De ahí que la posibilidad de cosechar sugiere la ausencia de contaminación.

De esta manera, en este conflicto, el discurso científico funciona como dispositivo de poder que produce cuerpos enfermos. Asi, para las y los vecinos esta construcción aparece como ficción, frente al despliegue de saberes "otros" que producen nuevos indicadores y parámetros. Se configura, entonces, la oposición experiencia-experimentación donde la primera refiere a aquello que "pasa" por el cuerpo, mientras que la experimentación es aquello que está por fuera, que permite ser observado mediante diversos métodos y procedimientos. En sintesis, el discurso científico emerge como dispositivo de poder en el caso de Pueblos Unidos ya que el argumento de la presencia de contaminación y cuerpos enfermos sustenta la voluntad de desalojar esas tierras.

Por su parte, en el conflicto Vudas contra Porta Hnos. el cuerpo enfermo asume un rol central en el conflicto. A diferencia de Pueblos Unidos, que discute la presencia de contaminación y la enfermedad del cuerpo emerge como ficción, en Vudas la enfermedad del cuerpo es una certeza y es desde alli que se inicia una lucha por el reconocimiento científico:

ER1: hace tres años que vivimos con el corazón
en la boca sin saber si te toca a vos el cáncer, si
te toca a vos y no a uno de los chicos, mirándolos
a los chicos en todo, si le salen sarpullidos o no,
si qué le va a pasar, por que el hijo de Gabriela
tiene púrpura y ya le jodieron la vida para siem-
pre con la púrpura. Escuchando hablar a la
gente que sabe, profesionales hablan de que l...]
edemas pulmonares y cáncer de pulmón, por
que todo lo que vos respiras, entra por dentro
y se va depositado y ya tenemos muertos de
cáncer de pulmón, cuatro muertos de cáncer
de páncreas, osea son indicadores, tenemos un
estudio presentado a Mana de la universidad de
los chicos de biologia en donde descubrieron
en las altas concentraciones de estomago, l...
ellos descubrieron las altas concentraciones
de dióxido de carbono. Entonces llegan a la
conclusión de que el lugar está contaminado
¿A donde esta el estudio? ¿Alguien le hizo caso
al estudio? No. y eso está presentado entonces
cómo podes pensar vos que estamos psicoló-
gicamente nosotros, cansados, que si se nos
baa, sabemos muy bien que si a nosotros se nos
bajan las defensas va a ser para que nos ataque
más rápidamente cualquier cosa (Vudas $54: 29$ ).

En el discurso de Vudas el cuerpo aparece como aquello expuesto a la contaminación, aquello que debe ser inspeccionado a cotidiano a los fines de encontrar alguna anomalía. La inspección asume sentido ya que se parte de la certeza de que la enfermedad es inminente y solo es una cuestión de tiempo saber a quién le toca. La contaminación tóxica es "inherentemente incierta" (Edelstein 2003). Las exposiciones pasadas del cuerpo, la relación dosis-respuesta, los efectos sinérgicos y la ambigüedad etiológica, todos contribuyen al problema de la ambigüedad tanto en la toxicología como en la epidemiología (Brown et al. 2000). Así, el cuerpo es amenazado, atacado y debe protegerse garantizando un buen sistema de defensas.

Las integrantes del colectivo en lucha cuentan con un conocimiento específico de las enfermedades que genera la exposición a los químicos. Esto se expresa en la cita en una descripción y 
enumeración de enfermedades posibles y su consiguiente recuento de casos en el barrio. El cuidadoso registro de las enfermedades que se han presentado resulta fundamental como "indicadores" de contaminación. El cuerpo es significado como afectado fruto de la exposición a la que han sido sometidos/as. La noción de afectados ambientales ${ }^{6}$ no solo refiere al impacto de los químicos en los cuerpos humanos sino que, desde una perspectiva sociológica designa el extenso y vital proceso de lucha por el que deben atravesar para lograr el reconocimiento de su afección. Supone un largo camino que se inicia desde la percepción del daño en sus cuerpos, hasta la visibilización en el espacio público: aparición, denuncia pública y judicial, movilización colectiva, interpelación a autoridades públicas para lograr el reconocimiento del daño y del riesgo, la sanción de los responsables, la afirmación y exigencia de una condena, la reparación y el resarcimiento a las víctimas (Berger 2016).

El concepto de afectados ambientales presenta una complejidad singular. Esta complejidad refiere a la relación entre la afección y la necesidad de reconocimiento público. En otras palabras, exige la vinculación y aceptación de que las actividades económicas en cuestión dañan la salud y son la causante principal de la aparición de diversas enfermedades que presentan los afectados. Esto, se vincula a su vez con una segunda complejidad: la vinculación entre las actividades y las enfermedades se encuentra mediatizada por el discurso científico. La sola afirmación de las y los afectados no ha sido suficiente para las autoridades, sino que han sido forzados a "comprobarlo", por lo cual el discurso científico ha sido depositario de un inmenso poder en el conflicto. En otras palabras, el cuerpo de las y los afectados solo se vuelve inteligible para el Estado a partir de la "traducción", interpretación y vinculación que puede realizar el discurso científico.

Las personas que sufren los efectos de la contaminación ambiental son en su totalidad victimas inocentes. No saben del problema al que están expuestas hasta que empiezan a percibir en sus propios cuerpos los impactos: afecciones dermatológicas, respiratorias, gastrointestinales, nerviosas, disrupciones endocrinas. Comienzan a indagar por sus propios medios sobre la toxicología que se relaciona con las actividades económicas con las que conviven - que identifican como probables causantes de esas afecciones y de otras más crónicas a la salud -, y descubren su vinculación con la proliferación de cánceres de todo tipo, malformaciones en fetos y abortos espontáneos y esterilidad masculina y femenina. Al hacerlo, descubren un mundo nuevo de términos, de especialidades médicas y de tipos de estudios ambientales (Berger 2016). Así, las y los afectados procuran dar cuenta de sus afecciones a partir del desarrollo de múltiples estudios científicos y relevamientos comunitarios que fundamentan sus denuncias.

No obstante, la respuesta del Estado, más que un acercamiento al reconocimiento, es una suma de dispositivos que profundizan la injusticia: invisibilización, abandono institucional, exclusión de la participación, discriminación y estigmatización. Así, el cuerpo, la experiencia y el testimonio de las y los afectados ponen en evidencia la omisión y falta de responsabilidades por parte del estado que hacen a la vulneración de derechos.

Las comunidades exigen procedimientos de elaboración de políticas que fomenten la participación comunitaria activa que institucionalicen la participación pública, que reconozcan al conocimiento comunitario, y utilicen formatos e intercambios interculturales para posibilitar la participación de tanta diversidad como exista en una comunidad. Los grupos de justicia ambiental reclaman un «lugar

\footnotetext{
6 Este concepto ha asumido gran relevancia en el proceso de lucha en Brasil, del Movimento de Atingidos pelas Barragens (Movimiento de Afectados por las Represas) ha permitido la adopción de una definición clara de afectado como parte de un proceso de definición de politicas y de evaluaciones de impacto ambiental, con un fuerte componente de institucionalización. En el contexto de disputas y evolución del concepto, el MAB logró el reconocimiento del afectado como inundado, entrando en consideración no solo una visión patrimonialista sino también la de los no propietarios, o aun cuando lo fuera, el afectado es el desplazado involuntariamente, visión en la que sigue predominando el derecho del emprendedor por sobre el derecho del afectado y la «hipostasis del agua como culpable» (Vainer 2012). Esta modificación dio lugar posteriormente a discutir los límites de la noción de impacto y la desestructuración de relaciones sociales que generan los emprendimientos: entender el proceso en términos no solamente materiales o pecuniarios (Santos 2015).
} 
en la mesa» y el derecho de "hablar por nosotros mismos" (Schlosberg 2010 en Berger 2016).

Así, se produce un hiato entre la autopercepción de las y los afectados sobre sus cuerpos y los diversos estudios científicos que presentan como pruebas y el discurso científico que se produce en el marco de la institucionalización de la lucha. En otras palabras, mientras que las y los vecinos cuentan con inumerables pruebas cientíicas que afirman la presencia de contaminación y su efecto en los cuerpos, la justicia y las instituciones los niegan, solicitan la realización de nuevos estudios científicos que, o bien nunca se realizan o incluso se llevan a cabo y desmienten la presencia de contaminación y la imposibilidad de vincular las actividades económicas con las afecciones. Este último argumento se basa en la multicausalidad de las enfermedades: ya que la aparición de enfermedades no puede vincularse con solo un factor, ello inhabilitaría ponderar cuáles son las causas principales.

En sintesis, en los discursos de este conflicto el cuerpo aparece como superficie de exposición, donde el sacrificio de algunos es preciso para el "avance" científico-tecnológico ya que el discurso científico ha sido cooptado por el poder.

\section{Reflexiones finales}

En este artículo hemos abordado los discursos científicos sobre la contaminación en dos conflictos por el ambiente en Córdoba, Argentina. En ambos casos estudiados los discursos científicos se configuran como dispositivos de poder por la relación que establecen con las diversas fracciones del capital y las efectuaciones que se producen en los cuerpos.

Los discursos científicos son discursos de verdad que pueden funcionar como dispositivos de poder de acuerdo a sus características:

a) Conexiones intimas entre saber y poder: en el caso de Pueblos Unidos el discurso científico sostiene la presencia irremediable de contaminación. Una gran parte de la comunidad barrial denuncia que la afirmación de contaminación se explica por el interés del capital inmobiliario que demanda la "liberación" del territorio a los fines de construir alli un emprendimiento. Así, el discurso científico como discurso de verdad expresa su poder al fundamentar la posible acción de despojo de la tierra a través del desalojo de la población.

Por su parte, en el caso de Vudas contra Porta Hnos. el discurso científico niega la presencia de contaminación y, consiguientemente, el vínculo con las enfermedades que se registran en el barrio. Las vecinas denuncian que el ocultamiento de la contaminación se debe a la protección de los intereses de la fábrica que se rehúsa a ser relocalizada. Así, el discurso científico como discurso de verdad expresa su poder al evitar el desalojo y relocalización de la fábrica.

b) Establecer la dispersión del poder a través de múltiples técnicas: las técnicas más importantes en los discursos analizados son los exámenes o pruebas científicas desarrolladas que, en ambos casos son impugnadas por las comunidades. En el caso de Pueblos Unidos, los vecinos ponen en duda los resultados ya que las muestras fueron recolectadas sin la presencia de la población, como se había acordado previamente, y, a su vez, los resultados de plomo en sangre fueron anunciados oralmente sin una justificación contundente.

Por su parte, en el caso de Vudas contra Porta Hnos. las vecinas denuncian la expresa manipulación de los resultados y el vínculo que comprometía al perito oficial con la empresa en cuestión.

En ambos casos, es la puesta en práctica del método científico aquella que se encuentra en cuestión e impugnada. Así, la manipulación de las técnicas científicas se relaciona en el discurso con el mecanismo que permite favorecer los intereses de los capitales.

c) Se trata de un conjunto heterogéneo que incluye diversos elementos: discursos, instituciones, edificios, leyes, medidas policiacas, proposiciones filosóficas: en ambos casos estudiados, el discurso científico se configura como dispositivo de poder que sirve de sustento y fundamento para activar/desactivar el accionar estatal. En 
el caso de Pueblos Unidos la afirmación de la presencia de contaminación puso en marcha diversos mecanismos del Estado que declararon "inhabitable" el territorio. Esta declaración tiene efectos concretos y cotidianos en las condiciones de vida posibles para la población ya que impide el acceso legal a los servicios básicos para la vida como el agua, luz y gas. En otras palabras, el dispositivo de poder del discurso científico se articula con una diversidad de mecanismos que efectúan condiciones que favorecen la expulsión de la población. Por su parte, en el caso de Vudas el dispositivo del discurso científico posibilita la inacción estatal y niega la necesidad de relocalización de la fábrica.

d) El dispositivo de poder siempre tiene una función concreta inscripta en las relaciones de poder: como se ha dicho, en ambos casos el dispositivo del discurso científico permite la acumulación de las fracciones del capital especulativo- inmobiliario y agro-industrial respectivamente. El poder del discurso científico habilita y legitima el desarrollo de estas actividades económicas.

De alli que los discursos científicos se desplieguen en los casos analizados como dispositivos de poder. Estos dispositivos efectúan corporalidades diversas en ambos casos:

- Los cuerpos libres de contaminación: en el conflicto de Pueblos Unidos el cuerpo es percibido como sano y la enfermedad aparece como una ficción producida por el discurso científico. Este es utilizado por el capital inmobiliario a los fines de producir ficciones que permitan el desalojo de esta población y luego construir allí un emprendimiento edilicio de alto valor. Si el discurso científico no es confiable el cuerpo debe encontrar/producir parámetros de análisis propios. La observación de la naturaleza resulta fundamental para conocer las condiciones del suelo. Si hay cosechas, la tierra se encuentra apta para la vida. Estos análisis que los sujetos realizan les permiten sostener la idea de que los cuerpos se encuentran libres de contaminación.

- Afectados ambientales: En el conflicto Vudas contra Porta Hnos. el dispositivo del discur- so científico se configura como aquello que impide dar cuenta del cuerpo enfermo. La temporalidad de la enfermedad aparece como actual, aunque silenciosa. De allí que el desarrollo de una estrategia para luchar en contra del dispositivo del discurso científico y apropiarlo resulte clave para este caso. El cuerpo asume la marca de la enfermedad, la reivindica y la constituye en denuncia. Los afectados ambientales son quienes han sido atacados por la contaminación y enfermados por ella. Para los afectados ambientales la única posibilidad es el reconocimiento público de la afección y la lucha por la defensa de la vida de quienes aún no han sido enfermados.

En sintesis, los discursos científicos en los casos analizados funcionan como dispositivos de poder que, de acuerdo a la articulación que establezcan con el capital, efectúan cuerpos singulares. En el caso de Pueblos Unidos, cuerpos sanos sobre los cuales se erige una ficción de enfermedad, mientras que en Vudas, cuerpos afectados por el ambiente.

Finalmente, restan indagar en futuras investigaciones los discursos del municipio y el de la empresa Porta Hnos. para conocer cómo significan la idea de contaminación, riesgo ambiental y las representaciones que configuran sobre el discurso científico a los fines de poder ponerlas en diálogo con las significaciones de las comunidades barriales.

\section{Referencias}

Auyero, Javier and Debora Swistun. 2008. The social production of toxic uncertainty. American Sociological Review, 73 (3): 357-379. https://doi. org/10.1177/000312240807300301.

Berger, Mauricio. 2016. Afectados ambientales: hacia una conceptualización en el contexto de luchas por el reconocimiento. Debates en Sociología 42: 31-53.

Berger, Mauricio. 2013. Cuerpo, experiencia, narración: auto-organización ciudadana en situaciones de contaminación ambiental. Córdoba: Ed. del Boulevard.

Brown, Phil, Steve Kroll- Smith and Valerie Gunter. 2000 Knowledge, citizens, and organizations: an overview of environments, diseases, and social conflict. In /llness and the environment: a reader in contested medicin, edited by Phil Brown, Steve Kroll-Smith y Valerie Gunter, 9-25. New York: New York University Press. 
Ciuffolini, Maria Alejandra. 2010. Resistencias: luchas sociales urbanas en Córdoba post-2001. Córdoba: Educc.

Deleuze, Gilles. 1994. Lógica del sentido. Barcelona: Paidós.

Douglas, Mary and Aaron Wildavsky. 1982. Risk and culture: an essay on the selection of technological and environmental dangers. California: University of California Press.

Edelstein, Michael. 2003. Contaminated communities: the social and psychological impacts of residential toxic exposure. Colorado: Westview Press.

Foucault, Michel. 1994. Dichos y escritos. Tomo III. 2 ed. Madrid: Editorial Nacional.

Guattari, Felix y Suely Rolnik. 2005. Micropolitica. Cartografias del deseo. Madrid: Traficantes de Sueños.

Lezama, José Luis. 2001. El medio ambiente como construcción social: reflexiones sobre la contaminación del aire en la Ciudad de México. Estudios Sociológicos 19 (56): 325-338.

Murillo, Susana. 2004. El nuevo pacto social, la criminalización de los movimientos sociales y la ideología de la seguridad. Revista Osal 14: 261-273.

Orellana, René. 1999. Conflictos ¿sociales, ambientales, socioambientales? Conflictos y controversias en la definición de conceptos. In Comunidades y conflictos socioambientales. experiencias y desafios en América Latina, editado por Pablo Ortiz, 331-344. Quito: Ediciones UPS Abya-Yala.

Sabatini, Francisco. 1997. Chile: conflictos ambientales locales y profundización democrática. Revista Ecología Politica 13: 51-70.

Saccucci, Erika. 2019. Estudio de la significación de la inseguridad ambiental en dos casos en Córdoba. Sustentabilidade em Debate 10 (1): 132-145. https://doi. org/10.18472/SustDeb.v10n1.2019.19137

Santos, Mariana dos. 2015. O conceito de "atingido" por barragens-direitos humanos e cidadania. Revista Direito e Práxis 6 (2): 113-140. https://doi.org/10.12957/ dep.2015.12698.

Vainer, Carlos. 2012. O concepto de atingido. In Justicia ambiental y creatividad democrática, editado por Cecilia Carrizo y Mauricio Berger, 95-118. Córdoba: Alción.

\section{Eríka Saccucci}

Licenciada en Ciencia Política y Doctora en Estudios Sociales de América Latina. Becaria posdoctoral Conicet radicada en el Área de Ciencias Sociales y Humanidades de la Universidad Católica de Córdoba (UCC), Córdoba, Argentina. Adjunta en Cátedra de Epistemología de las Ciencias Sociales. Miembro del Colectivo de Investigación el Llano en Llamas. 